\title{
Synthesis and Characterization of Crystalline
}

\section{Hyperbranched Polysiloxysilane with POSS Groups at}

\section{the Terminal Position}

Makoto Seino, Teruaki Hayakawa, Yoshihito Ishida, and Masa-aki Kakimoto*

Department of Organic and Polymeric Materials, Tokyo Institute of Technology, O-okayama, Meguro-ku, Tokyo 152-8550, Japan

*Corresponding author

E-mail: mkakimot@o.cc.titech.ac.jp

Tel: +81-3-5734-2433

Fax: +81-3-5734-2875

Materials. $A n \mathrm{AB}_{2}$ monomer, 1,5-divinyl-1,1,3,5,5-pentamethyltrisiloxane (Kozima Chemical Inc., Japan), was purified by distillation. Platinum 1,3-divinyl-1,1,3,3-tetramethyldisiloxane complex $(\operatorname{Pt}(\mathrm{dvs})) \quad 0.1 \mathrm{M}$ solution in xylene and hydro-heptacyclopentyl substituted polyhedral oligomeric silsesquioxane (POSS-H) were purchased from Aldrich. Toluene was distilled with sodium.

Measurements. ${ }^{1} \mathrm{H},{ }^{13} \mathrm{C}$ and ${ }^{29} \mathrm{Si}$ NMR spectra were recorded in $\mathrm{CDCl}_{3}$ on a JEOL JNM-AL 300 spectrometer. IR spectra were recorded on a JASCO FT/IR-460 Plus spectrophotometer. Molecular weights were determined by gel permeation chromatography (GPC) using a Shodex GPC-101 system with Minidawn (Wyatt Technology) as light scattering detector and Shodex KF-803L and 804L columns 
using THF as an eluent. Differential scanning calorimetry (DSC) was measured with a Seiko DSC 6200 at a heating rate of $10{ }^{\circ} \mathrm{C} / \mathrm{min}$ under nitrogen. Micrographs were taken on an Olympus BX51. Wide-angle X-ray patterns were observed using a Rigaku-Denki X-ray generater.

HBPS-Vi. In a $100 \mathrm{~mL}$ flask, $\mathrm{Pt}(\mathrm{dvs})$ solution $(0.1 \mathrm{M}$ in xylene) $(0.5 \mathrm{~mL}, 0.05 \mathrm{mmol})$ was added to 1,5-divinyl-1,1,3,5,5-pentamethyltrisiloxane (12.327 g, $0.05 \mathrm{~mol})$ under argon, and the reaction mixture was stirred at room temperature for $3 \mathrm{~h}$. After reprecipitation from diethyl ether into methanol, the precipitate was collected and dried in vacuum to give HBPS-Vi $(5.964 \mathrm{~g}$, yield $48 \%) . M_{\mathrm{w}}=9,300$, $M_{\mathrm{w}} / M_{\mathrm{n}}=2.0$ (GPC). $T_{\mathrm{g}}:-99{ }^{\circ} \mathrm{C}(\mathrm{DSC}) .{ }^{1} \mathrm{H} \mathrm{NMR}: \delta-0.09$ (br, $\left.\mathrm{Si}\left(\mathrm{CH}_{3}\right) \mathrm{CHSi}\right),-0.02-0.09(\mathrm{~m}, \mathrm{SiCH})$, 0.13-0.18 (m, OSi $\left.\left(\mathrm{CH}_{3}\right)_{2} \mathrm{CH}=\mathrm{CH}_{2}\right), 0.42$ (br, $\left.\mathrm{SiC}_{2} \mathrm{H}_{4} \mathrm{Si}\right), 0.91-1.10$ (m, Si(CH3)CHSi), 5.66-5.93 (m, $\left.\mathrm{SiCH}=\mathrm{CH}_{2}\right), \quad 6.04-6.17 \quad\left(\mathrm{~m}, \quad \mathrm{SiCH}=\mathrm{CH}_{2}\right) .{ }^{13} \mathrm{C} \quad \mathrm{NMR}: \quad \delta-1.10-1.63 \quad\left(\mathrm{~m}, \quad \mathrm{SiCH}_{3}\right), \quad 7.92-8.22 \quad(\mathrm{~m}$, $\left.\mathrm{Si}\left(\mathrm{CH}_{3}\right) \mathrm{CHSi}\right), 8.85-9.11$ (m, $\left.\mathrm{SiCH} \mathrm{CH}_{2}\right), 9.40-9.75$ (m, $\left.\mathrm{SiCH}\right)$, 11.84-12.05 (m, $\left.\mathrm{Si}\left(\mathrm{CH}_{3}\right) C H \mathrm{Si}\right), 131.61$ (s, $\left.\mathrm{SiCH}=\mathrm{CH}_{2}\right), 139.41-139.48\left(\mathrm{~m}, \mathrm{SiCH}=\mathrm{CH}_{2}\right) .{ }^{29} \mathrm{Si} \mathrm{NMR}: \delta-21.79--19.71\left(\mathrm{~m}, \mathrm{CSiMeO}_{2}\right),-4.32(\mathrm{br}$, $\mathrm{OSiMe}_{2} \mathrm{CH}=\mathrm{CH}_{2}$ ), 6.40-8.60 (br, CSiMe $2 \mathrm{O}$ ). IR (neat, $\mathrm{cm}^{-1}$ ): 3051, 2958, 2910, 2874, 1407, 1256, 1135, $1046,1009,955,837,783,703$.

P1. To a $20 \mathrm{~mL}$ Schlenk flask were added HBPS-Vi (0.074 g, $0.3 \mathrm{mmol})$, POSS-H (0.361 g, 0.4 mmol) and toluene ( $3 \mathrm{~mL})$ under Argon. Pt(dvs) $(20 \mu \mathrm{L})$ was added, and the mixture was stirred at 100 ${ }^{\circ} \mathrm{C}$ for $24 \mathrm{~h}$. After POSS-H (0.361 g, $\left.0.4 \mathrm{mmol}\right)$, toluene $(2 \mathrm{~mL})$ and $\mathrm{Pt}(\mathrm{dvs})(20 \mu \mathrm{L})$ were added, the mixture was stirred for additional $48 \mathrm{~h}$. The products were isolated by preparative GPC. Removal of the solvent gave P1 (white solid, $0.200 \mathrm{~g}$, yield $58 \%$ ). $M_{\mathrm{w}}=24,300, M_{\mathrm{w}} / M_{\mathrm{n}}=1.4$ (GPC). $T_{\mathrm{g}}: 18{ }^{\circ} \mathrm{C}(\mathrm{DSC})$. $T_{\mathrm{m}}: 233{ }^{\circ} \mathrm{C}$ (DSC). ${ }^{1} \mathrm{H}$ NMR: $\delta-0.15$ (br, $\mathrm{Si}\left(\mathrm{CH}_{3}\right) \mathrm{CHSi}$ ), -0.03-0.07 (br, $\mathrm{SiCH}$ ), 0.30-0.60 (br, $\mathrm{SiC}_{2} \mathrm{H}_{4} \mathrm{Si}$ ), 0.85-1.05 (br, cyclopentyl $\mathrm{CH}$ ), 1.35-1.80 (m, cyclopentyl $\left.\mathrm{CH}_{2}\right) .{ }^{13} \mathrm{C}$ NMR: $\delta$-1.21-1.53 (m, $\mathrm{SiCH}_{3}$ ), 3.78 (br, $\mathrm{CH}_{2} \mathrm{CH}_{2} \mathrm{POSS}$ ), 7.88 (br, $\mathrm{Si}\left(\mathrm{CH}_{3}\right) \mathrm{CHSi}$ ), 9.00 (br, $\mathrm{SiCH}$ ), 9.30 (br, $\mathrm{CH}_{2} \mathrm{CH}_{2} \mathrm{POSS}$ ), 9.53 (br, $\mathrm{SiCH}$ ), 11.84 (br, $\mathrm{Si}\left(\mathrm{CH}_{3}\right) C H \mathrm{Si}$ ), 22.22 (s, cyclopentyl $\mathrm{CH}$ ), 26.98, 27.01 (2s, cyclopentyl $\mathrm{CH}_{2}$ ), 27.27, 27.31 (2s, cyclopentyl $\left.\mathrm{CH}_{2}\right) .{ }^{29} \mathrm{Si} \mathrm{NMR}: \delta-66.80--66.08$ (m, POSS) -21.73- -21.12 (m, $\left.\mathrm{CSiMeO}_{2}\right), 7.89$ (br, CSiMe $\left.2 \mathrm{O}\right)$. IR $\left(\mathrm{KBr}, \mathrm{cm}^{-1}\right)$ : 2952, 2867, 1452, 1408, 1255, 1112, 1046, 915, 838, 
782.

P2 (white solid, $0.210 \mathrm{~g}$, yield $75 \%$ ) was prepared from hydrosilylation of HBPS-Vi (0.4 mmol, $0.099 \mathrm{~g})$ with POSS-H $(0.2 \mathrm{mmol}, 0.180 \mathrm{~g})$ similarly. $M_{\mathrm{w}}=19,800, M_{\mathrm{w}} / M_{\mathrm{n}}=1.7(\mathrm{GPC}) . T_{\mathrm{g}}: 16{ }^{\circ} \mathrm{C}$ (DSC). $T_{\mathrm{m}}: 211{ }^{\circ} \mathrm{C}$ (DSC). ${ }^{1} \mathrm{H}$ NMR: $\delta$-0.11 (br, $\mathrm{Si}\left(\mathrm{CH}_{3}\right) \mathrm{CHSi}$ ), -0.02-0.13 (br, $\mathrm{SiCH}_{3}$ ), 0.30-0.60 (br, $\mathrm{SiC}_{2} \mathrm{H}_{4} \mathrm{Si}$ ), 0.92-1.00 (br, cyclopentyl $\mathrm{CH}$ ), 1.40-1.80 (m, cyclopentyl $\left.\mathrm{CH}_{2}\right) .5 .66-5.91\left(\mathrm{~m}, \mathrm{SiCH}=\mathrm{CH}_{2}\right)$, 6.04-6.15 (m, $\mathrm{SiCH}=\mathrm{CH}_{2}$ ). ${ }^{13} \mathrm{C}$ NMR: $\delta$-1.17-1.57 (m, $\mathrm{SiCH}_{3}$ ), 3.80 (br, $\mathrm{CH}_{2} \mathrm{CH}_{2} \mathrm{POSS}$ ), 7.89 (br, $\mathrm{Si}\left(\mathrm{CH}_{3}\right) \mathrm{CHSi}$ ), 8.92 (br, $\mathrm{SiCH}$ ), 9.33 (br, $\mathrm{CH}_{2} \mathrm{CH}_{2} \mathrm{POSS}$ ), 9.56 (br, $\mathrm{SiCH}$ ), 11.84 (br, m, $\left.\mathrm{Si}\left(\mathrm{CH}_{3}\right) C H S i\right)$, 22.24 (s, cyclopentyl $\mathrm{CH}$ ), 27.01 (s, cyclopentyl $\mathrm{CH}_{2}$ ), 27.28, 27.32 (2s, cyclopentyl $\mathrm{CH}_{2}$ ), 131.54 (s, $\left.\mathrm{SiCH}=\mathrm{CH}_{2}\right), 139.41\left(\mathrm{~m}, \mathrm{SiCH}=\mathrm{CH}_{2}\right) .{ }^{29} \mathrm{Si} \mathrm{NMR}: \delta-66.72--65.92(\mathrm{~m}, \mathrm{POSS})-21.06$ (br, $\mathrm{CSiMeO}_{2}$ ), -4.31 (br, $\left.\mathrm{OSiMe}_{2} \mathrm{CH}=\mathrm{CH}_{2}\right), 8.04$ (br, CSiMe $\left.2 \mathrm{O}\right) . \mathrm{IR}\left(\mathrm{KBr}, \mathrm{cm}^{-1}\right):$ 2952, 2867, 1452, 1407, 1256, 1110, $1046,954,915,837,783$.

P3 (white solid, $0.299 \mathrm{~g}$, yield $70 \%$ ) was prepared from hydrosilylation of HBPS-Vi (1 mmol, $0.247 \mathrm{~g})$ and POSS-H $(0.2 \mathrm{mmol}, 0.180 \mathrm{~g})$ similarly. $M_{\mathrm{w}}=15,600, M_{\mathrm{w}} / M_{\mathrm{n}}=1.7(\mathrm{GPC}) . T_{\mathrm{g}}: 18{ }^{\circ} \mathrm{C}(\mathrm{DSC})$. $T_{\mathrm{m}}: 167{ }^{\circ} \mathrm{C}$ (DSC). ${ }^{1} \mathrm{H}$ NMR: $\delta$-0.10 (br, $\mathrm{Si}\left(\mathrm{CH}_{3}\right) \mathrm{CHSi}$ ), -0.02-0.18 (br, $\mathrm{SiCH}$ ), 0.36-0.57 (br, $\mathrm{SiC}_{2} \mathrm{H}_{4} \mathrm{Si}$ ), 0.95-1.01 (br, cyclopentyl $\mathrm{CH}$ ), 1.47-1.73 (m, cyclopentyl $\left.\mathrm{CH}_{2}\right)$. 5.66-5.93 (m, $\mathrm{SiCH}=\mathrm{CH}_{2}$ ), 6.04-6.17 (m, $\left.\mathrm{SiCH}=\mathrm{CH}_{2}\right) .{ }^{13} \mathrm{C}$ NMR: $\delta$-1.16-1.59 (m, $\left.\mathrm{SiCH}_{3}\right), 3.83\left(\mathrm{~m}, \mathrm{CH}_{2} \mathrm{CH}_{2} \mathrm{POSS}\right.$ ), 7.91 (br, $\mathrm{Si}\left(\mathrm{CH}_{3}\right) \mathrm{CHSi}$ ), 8.92 (br, $\mathrm{SiCH}$ ), 9.41 (br, $\mathrm{CH}_{2} \mathrm{CH}_{2} \mathrm{POSS}$ ), 9.56 (br, $\mathrm{SiCH}$ ), 11.82 (br, br, $\mathrm{Si}\left(\mathrm{CH}_{3}\right) C H S i$ ), 22.23 (s, cyclopentyl $\mathrm{CH}$ ), 26.98 (s, cyclopentyl $\mathrm{CH}_{2}$ ), 27.01, 27.32 (2s, cyclopentyl $\mathrm{CH}_{2}$ ), 131.58 (s, $\left.\mathrm{SiCH}=\mathrm{CH}_{2}\right), 139.41\left(\mathrm{~m}, \mathrm{SiCH}=\mathrm{CH}_{2}\right) .{ }^{29} \mathrm{Si} \mathrm{NMR}: \delta-66.72--65.92(\mathrm{~m}, \mathrm{POSS})-21.06\left(\mathrm{br}, \mathrm{CSiMeO}_{2}\right)$, -4.31 (br, $\left.\mathrm{OSiMe}_{2} \mathrm{CH}=\mathrm{CH}_{2}\right), 8.04$ (br, CSiMe $\left.2 \mathrm{O}\right) . \mathrm{IR}\left(\mathrm{KBr}, \mathrm{cm}^{-1}\right)$ : 2953, 2868, 1450, 1407, 1256, 1105, $1047,954,915,837,784$. 

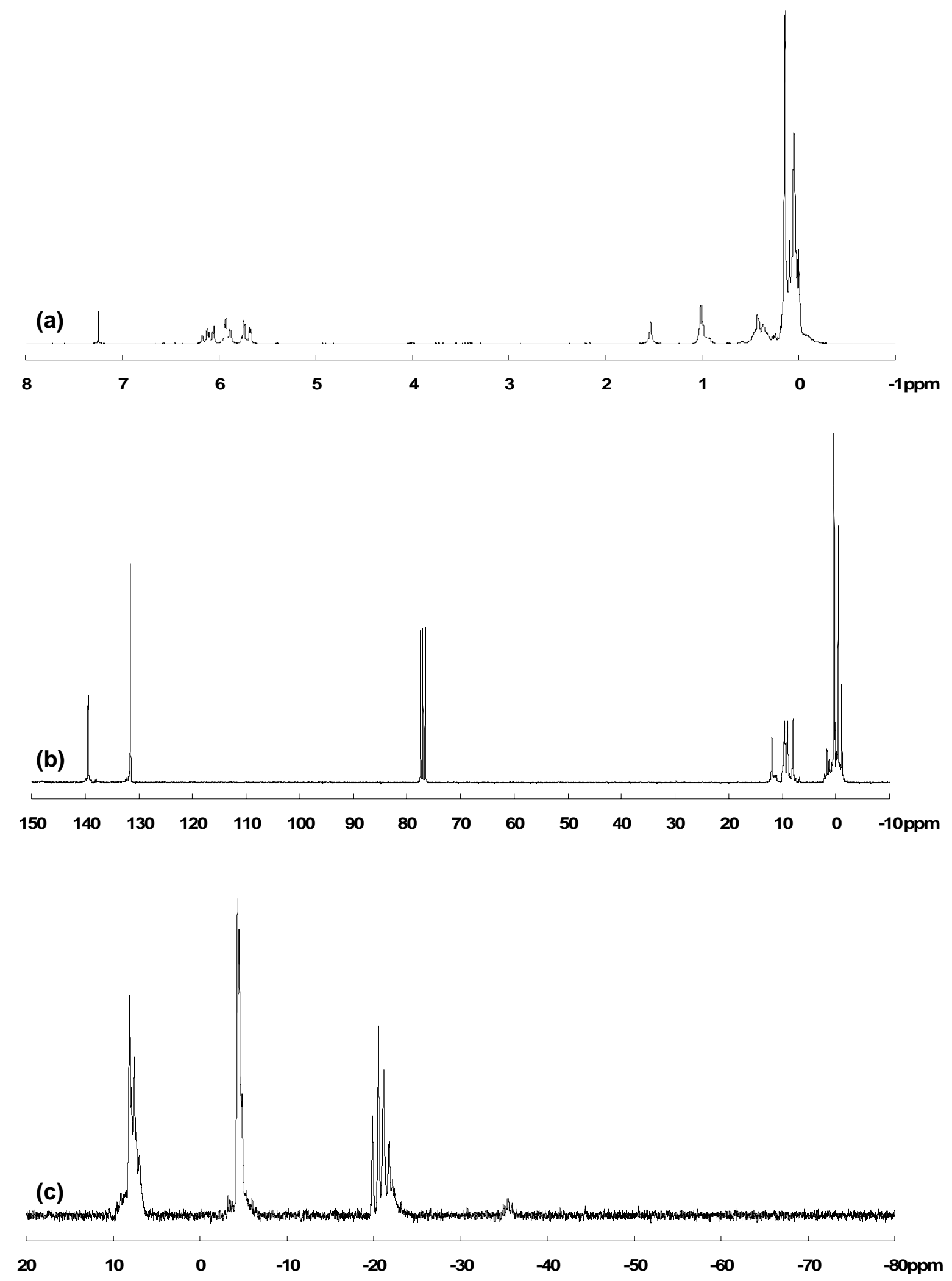

Figure 1. ${ }^{1} \mathrm{H}(\mathrm{a}),{ }^{13} \mathrm{C}$ (b), and ${ }^{29} \mathrm{Si}$ (c) NMR spectra of HBPS-Vi. 

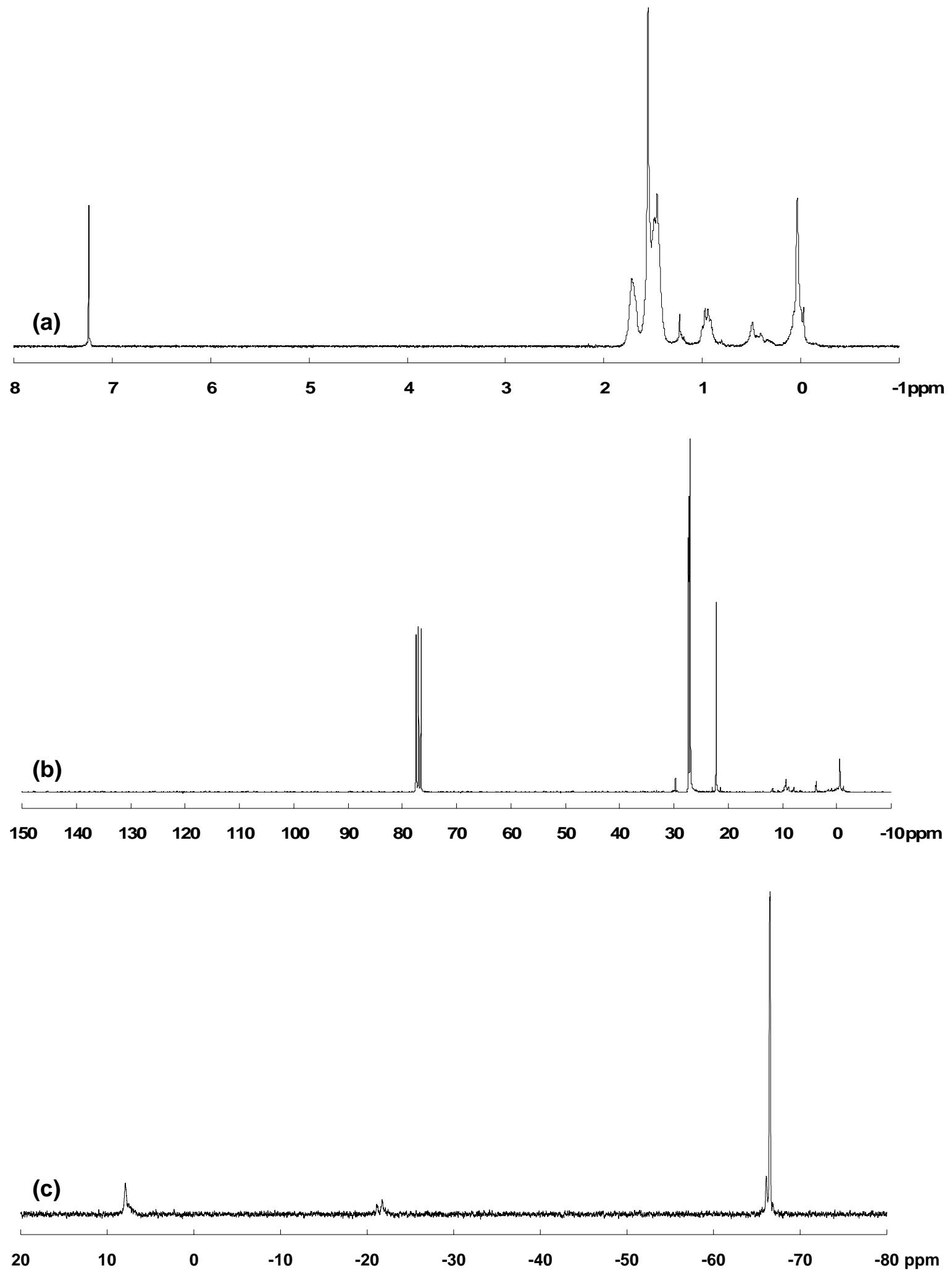

Figure 2. ${ }^{1} \mathrm{H}(\mathrm{a}),{ }^{13} \mathrm{C}(\mathrm{b})$, and ${ }^{29} \mathrm{Si}(\mathrm{c}) \mathrm{NMR}$ spectra of $\mathbf{P 1}$. 